\title{
Computerized Decision Support Systems for Mechanical Ventilation in Children
}

\author{
Katherine A. Sward ${ }^{1} \quad$ Christopher J. L. Newth ${ }^{2}$ \\ 1 Department of Biomedical Informatics, College of Nursing, \\ University of Utah, Salt Lake City, Utah, United States \\ 2 Department of Anesthesiology and Critical Care Medicine, Children's \\ Hospital Los Angeles and Keck School of Medicine, University of \\ Southern California, United States
}

J Pediatr Intensive Care 2016;5:95-100.

\begin{abstract}
Address for correspondence Katherine A. Sward, PhD, RN, Department of Biomedical Informatics, College of Nursing, University of Utah, 10 S 2000 East, Salt Lake City UT 84112, United States (e-mail: kathy.sward@nurs.utah.edu).
\end{abstract}

\begin{abstract}
Keywords

- mechanical ventilation

- computerized decision support

Mechanical ventilation is an effective treatment in the ICU but can have significant adverse effects. Approaches from adult research have been adopted in pediatric critical care despite known differences in respiratory physiology and ICU processes. There continues to be considerable variation in how ventilators are managed. Computerized decision support systems implement explicit protocols, and are designed to make mechanical ventilation management safer, more consistent, and more lung protective. Variable results and low or unknown compliance with protocols and CDSS tools have been reported. To date, there has been limited research regarding CDSS for mechanical ventilation in children.
\end{abstract}

\section{Introduction}

Mechanical ventilation is one of the most common lifesaving interventions in the intensive care unit (ICU), supporting approximately $30 \%$ of pediatric ICU (PICU) patients. ${ }^{1,2}$ Mortality caused by mechanical ventilation is relatively low, ${ }^{3}$ but the intervention is not without risk. Mechanical ventilation is known to cause lung injury even in the healthy lungs and contribute to further lung injury in the diseased lungs. Despite having a long history as an effective intervention, ${ }^{4}$ ventilators continue to evolve and little is known about how best to ventilate patients with specific conditions. ${ }^{3}$ There has been some progress in identifying optimal ventilator management approaches. The development of lung-protective ventilator strategies ${ }^{4}$ was of particular importance, having been refined since the description of protective benefits from permissive hypercapnia ${ }^{5}$ and the landmark ARDSNet publication in the New England Journal of Medicine ${ }^{6}$ showing benefit from lower tidal volumes. Other protective ventilator management strategies that have also shown promise include neuromuscular blockade ${ }^{7}$ and higher levels of positive endexpiratory pressure (PEEP) for certain subgroups of adult patients with acute respiratory distress syndrome (ARDS), ${ }^{8}$ as well as nonventilator interventions such as prone positioning, decreased sedation, and others. ${ }^{4,9,10}$

Over the past few years most pediatric intensivists have come to target tidal volumes of less than $8 \mathrm{~mL} / \mathrm{kg}^{1,11}$ based on the adult studies. However, the applicability of a low tidal volume strategy in pediatrics has never been formally and rigorously evaluated, especially since many pediatric practitioners favor pressure control modes with lung-protective pressures targeted rather than volumes. ${ }^{12}$ Additionally, factors such as time on the ventilator or readiness for extubation are common surrogate outcome measures in PICU studies. Without consistent ventilator management, ventilator-related outcome measures are subject to variability between centers and practitioners, which can obscure the effect of the intervention being investigated. ${ }^{13}$

It has become imperative that clinicians use information technology. Computerized decision support system (CDSS) tools offer a method to implement evidence-based processes, including complex guidelines and protocols, in a consistent manner. ${ }^{14}$ Most studies of mechanical ventilation CDSS tools have been done on adults, with only a small received

July 8,2015

accepted after revision

July 10, 2015

published online

November 18, 2015
Issue Theme Databases and

Computerized Systems in PICU;

Guest Editor: Philippe Jouvet, MD, PhD
DOI http://dx.doi.org/ 10.1055/s-0035-1568161. ISSN 2146-4618.
Copyright $\odot 2016$ by Georg Thieme Verlag KG, Stuttgart · New York 
handful involving children. As a result, many pediatric intensivists extrapolate conclusions from the adult studies. ${ }^{13}$ Yet, the respiratory system is physiologically different between infants, small children, and adults. ${ }^{15}$ Here, we review literature regarding CDSS in the PICU, beginning with broad observations about CDSS tools for mechanical ventilation in children, then examining a few specific areas of application. We focused on acute management of ventilators in pediatric intensive care and excluded literature regarding chronic, long-term ventilator use.

\section{CDSS Tools Affect Compliance with Explicit Protocols}

An agreed-upon protocol or guideline may improve consistency and consensus within and between PICUs. ${ }^{16}$ The ARDSNet ("lung protective") strategy has become a widely accepted approach for adult patients with acute lung injury (ALI) or ARDS. While the general concepts of the ARDSNetbased mechanical ventilation protocols have been accepted in pediatric practice, there appear to be several differences in application, particularly of PEEP (with pediatricians being more conservative than adult intensivists) and with the definition of extubation readiness. ${ }^{17,18}$ Despite a fairly long history of using PEEP, which has been demonstrated to improve outcomes in ALI and ARDS, the precise approach to choosing PEEP levels remains uncertain. ${ }^{4,19}$ Neither in children nor in adults has the optimal level of applied PEEP been determined. The PEEP/FIO 2 tables in the ARDSNet-based protocols are somewhat arbitrary and may result in undertreatment for some severe ARDS patients but may lead to overinflation in others. ${ }^{8}$ Insufficient pressure may not sufficiently support oxygenation, while excessive pressures can cause barotrauma. 4

A protocol or guideline is designed to guide decisions, based on knowledge regarding physiology, clinical trials and other evidence, and the expertise of experienced clinicians. Guidelines tend to be the most broad, often expressing principles without implementation details, whereas protocols tend to be more specific and describe actions to implement the guidelines. However, many protocols continue to need some interpretation and are often not specific enough to generate consistent decisions at the bedside. Significant variability in how the protocols are applied can still exist, ${ }^{20,21}$ and clinical data show that there continues to be wide variability in ventilator management. ${ }^{17,20,22}$ An explicit protocol provides enough detail to generate consistent recommendations, while individualization is preserved because each recommendation is driven by the patient's unique set of clinical data (patient state). ${ }^{20-23}$ Given the large number of potential clinical situations and responses, computers rapidly become useful for managing explicit protocols.

Two pediatric studies used paper protocols. Willson et $\mathrm{al}^{24}$ used a paper protocol outlining a broadly defined lungprotective strategy. Curley et $\mathrm{al}^{25}$ used the adult ARDSNet ventilation paper protocol. Neither paper addressed protocol compliance. The paper-based mechanical ventilation protocol used by the adult ARDSNet investigators was abstracted from an explicit computer protocol (CDSS) developed over a decade. ${ }^{23}$ Detailed paper-based protocols rapidly become lengthy and can be difficult to navigate. Literature in both adults and children has reported variable effects from mechanical ventilation protocols, ${ }^{20}$ with low ${ }^{26,27}$ or unknown ${ }^{14}$ adherence rates (protocol compliance), particularly for paperbased protocols. We still do not know with accuracy how children are mechanically ventilated in most PICUs. ${ }^{3}$ Implementing protocols on a computer as a CDSS provides consistent protocol navigation and interpretation of the protocol rules. The CDSS can track information about adherence to specific aspects of the protocol. ${ }^{20,21}$ High adherence has been demonstrated with adult ventilator CDSS tools, ${ }^{23,28}$ and preliminary data suggest that adherence will be reasonably high with pediatric ventilator CDSS tools. ${ }^{29}$ Studies in adult ICUs support the benefits of protocol-based ventilator management. Reported benefits included decreased duration and costs of mechanical ventilation and improved collaboration between health care team members.

\section{CDSS Knowledge Development}

In the complex environment that surrounds mechanical ventilation management in the PICU, explicit protocols implemented within a CDSS are important to limit unnecessary interclinician variability. ${ }^{22,30}$ The knowledge base ("rules") within an explicit mechanical ventilation CDSS needs to be extensive and detailed to capture the rich nuances of critically ill children. ${ }^{31}$ A major concern is obtaining agreement on what medical knowledge to implement within the CDSS. ${ }^{20}$ Published work on respiratory physiology, observational studies of ventilator management strategies and current practices, clinical trials, consensus conference findings, and expert clinician knowledge need to be synthesized to define decision points. ${ }^{13,20,21,30}$

Current CDSS for mechanical ventilation in pediatrics seem to be of two broad types. One type is commercial CDSS built into specific ventilators. Those commercialized CDSS tools are proprietary to the vendor and do not typically have mechanisms to modify or adapt the rules or reporting systems to capture reason for refusal of recommendations. ${ }^{20}$ Other CDSS tools are designed to be ventilator agnostic, but may be limited to particular modes of ventilation (e.g., pressure support or high-frequency oscillatory ventilation modes) or specific phases of the ventilation course (e.g., initiation of the ventilator, or the weaning phase).

Although pediatric intensivists claim to embrace evidence-based lung-protective strategies, the adherence to these strategies is often poor and the execution of lungprotective principles differs among individual practitioners. Explicit ventilator protocols offer the opportunity to standardize mechanical ventilation for children, if practitioners follow protocol recommendations ${ }^{10,13,22,32,33}$; CDSS tools can assist practitioners to adhere to the protocol.

Investigators in the PALISI and CPCCRN networks have been designing a CDSS to support lung-protective ventilation strategies in the PICU. ${ }^{20,22}$ This CDSS was derived from the adult ARDSNet protocols and CDSS tools, which have 
demonstrated effectiveness over many years. ${ }^{23,28,30,34}$ Little is known about the use in pediatric medicine of CDSS tools that were derived from adult tools. Success with an insulinmanagement CDSS tool simultaneously deployed in adult and pediatric ICUs suggest that such translation is possible. ${ }^{34,35}$ Appropriate translation from adult practices must be demonstrated rather than presumed, however. Given differences in respiratory physiology between adult and children, ${ }^{1,13,15}$ it is unlikely that a ventilator protocol developed in the adult ICU can simply be deployed in the pediatric setting. The protocol will likely need to be adapted, and must be shown to be safe, effective, and appropriately sized.

Clinicians need to be involved in developing and validating CDSS content/rules. ${ }^{13,20,21,30,36}$ Medical knowledge and ventilator technologies are constantly changing; the ease with which CDSS knowledge can be upgraded or adjusted to accommodate these changes could be a key factor in making mechanical ventilation CDSS widely accepted. ${ }^{20,37}$ Significant progress has been made in obtaining clinical consensus about how to manage ventilators for pediatric ALI/ARDS. An international consensus conference of experts in pediatric ALI/ARDS met over 2 years to develop agreed-upon definitions and recommendations for treatment, as well as to determine future research priorities and identify areas in need of further clarification. ${ }^{19}$ These recommendations are being incorporated into the PALISI/CPCCRN investigator's pediatric mechanical ventilation CDSS tools.

\section{Beyond if...then Rules}

Computers allow the CDSS to do more than apply "if...then" rules. ${ }^{20}$ The use of graphical displays to inform clinical decision making for mechanical ventilation was discussed by Bhutani. ${ }^{38}$ He graphically displayed pulmonary mechanics but did not provide explicit advice regarding how to manage the ventilators.

Complex calculations and physiologic equations can be incorporated into CDSS. ${ }^{20} \mathrm{~A}$ few adult ventilator CDSS have been reported, which accommodate uncertainty, using conditional probability and Bayesian logic $\mathrm{c}^{39,40}$ or fuzzy reasoning. ${ }^{41}$ Use of physiologic simulators has been proposed as a means to shorten development and validation times. Virtual patients with realistic responses could be used to develop and validate portions of the CDSS knowledge base. ${ }^{20}$ Several groups have begun work in this area, although no such platform was identified that is, as yet, widely available. ${ }^{42-47}$

One likely difference between pediatric and adult ventilator management practices lies in the former's reliance on noninvasive but continuous "blood gas" measurements over the adult intermittent measurement of arterial blood gas data. For example, pediatric intensivists more often monitor $\mathrm{Spo}_{2}$ over $\mathrm{PaO}_{2}{ }^{1,48}$ In the absence of arterial blood gas data, other parameters also need to be estimated. There has been promising evidence using $\mathrm{Petco}_{2}$ over $\mathrm{PaCO}_{2}$ as a surrogate for pH changes. ${ }^{48,49}$

\section{Closed-Loop CDSS}

The recommendations for changes to ventilator settings can be presented in either an open- or closed-loop manner. When the CDSS recommendation is acted upon without caregiver intervention, the recommendation is closed-loop. A recommendation is open-loop if it is displayed on the computer screen, with caregivers then deciding whether to act on the recommendation. However, clinical expertise and resources are not constantly available at the bedside. Clinicians typically adjust ventilator settings only every 8 hours, although the precise timing is highly variable. ${ }^{22,50,51}$ The ability to make frequent (breath-by-breath or minute-by-minute) adjustments to the ventilator according to the patient's condition (closed loop), with little or no caregiver intervention, could improve timeliness of ventilator changes and reduce clinician-induced variability. ${ }^{20} \mathrm{~A}$ closed-loop CDSS may also have a combination of open- and closed-loop components. ${ }^{20}$

The literature shows an increasing trend toward closedloop mechanical ventilation CDSS, also described as automation of mechanical ventilation. In the early 1990s, a group in the Netherlands ${ }^{52}$ evaluated an automated method for ventilator initiation; a computer algorithm determined the initial ventilator settings, as an exploration into the potential for future closed-loop studies. They found that the automated settings did not differ significantly from the settings determined by conventional method, that is, as determined by an intensive care professional. While settings were not found to be superior to conventional methods, the automated method required less manual data entry. Claure et al examined a mode called computer-assisted control of minute ventilation (CCMV) in preterm infants. ${ }^{53}$ This mode adjusts the ventilator rate, in response to spontaneous breaths and pulmonary mechanics. They found that CCMV maintained adequate oxygenation and ventilation with lower levels of ventilator support, compared with intermittent mandatory ventilation (IMV) mode.

In 2007 Jouvet et al used a closed-loop system to wean 20 children from mechanical ventilation. In this pilot/feasibility study the closed-loop protocol led to successful weaning and slightly shorter duration of mechanical ventilation, without increased need for reintubation. ${ }^{26}$ Recently, Jouvet and colleagues reported on a closed-loop method of weaning that adjusts support on a breath-by-breath basis, called adaptive support ventilation (ASV), in various configurations, with promising findings that warrant further investigation. ${ }^{54} \mathrm{~A}$ recent survey of PICU staff in the United Kingdom suggested that automated or closed-loop systems were available in $18 \%$ of their ICUs. ${ }^{55}$ Systematic reviews by Rose and Colleagues in 2012 and $2013^{56}$ compared studies of automated and nonautomated tools for weaning. They noted that the number of studies was limited and there was substantial heterogeneity in trial designs, but that the findings suggested that such systems may reduce weaning time, duration of ventilation, and duration of ICU stay, particularly in mixed or medical ICU populations.

Closed-loop systems appear promising, but more research needs to be done before they will become widespread. A completely closed-loop protocol could raise safety concerns among clinicians, which might reduce acceptability of the overall CDSS. There are often valid clinical reasons for declining CDSS instructions ${ }^{28,37}$ and 
there are several aspects of mechanical ventilation for which consensus has not yet been achieved. ${ }^{19}$ For example, there is little consensus about the management of PEEP. In patients with high pleural pressure on conventional ventilator settings, underinflation may lead to atelectasis, hypoxemia, and exacerbation of lung injury through "atelectrauma." In such patients, raising PEEP to maintain a slightly positive transpulmonary pressure might improve aeration and oxygenation without causing overdistension. Conversely, in patients with low pleural pressure, maintaining a low PEEP would keep transpulmonary pressure low, avoiding overdistension and consequent "volutrauma." Thus, the current strategy of setting PEEP without regard to transpulmonary pressure can be predicted to benefit some patients while harming others.

It seems reasonable to initially develop and validate CDSS in a predominantly open-loop manner, carefully evaluating reasons for declining recommendations and determining whether the knowledge within the CDSS needs to be adapted, and then moving toward progressively closed-loop for aspects in which the knowledge is well validated. ${ }^{20,30,36,37}$

\section{Early CDSS in Neonates}

Some of the early mechanical ventilation CDSS publications focused on neonates. In the 1980 s, Carlo et $\mathrm{al}^{57}$ reported on computer-assisted management of respiratory failure in neonates. Arterial blood gas values were more frequently improved in the computer-managed group, compared with when ventilators were managed without the computer program, and there were fewer events in which ventilator support was increased in the presence of normal blood gas values. Claure et al reported on a closed-loop CDSS used in 15 low-birth-weight, preterm infants, ${ }^{53}$ showing adequate ventilation and oxygenation with lower mechanical ventilator support, and suggested this might reduce risk for barotrauma or other ventilator-associated lung injury. Another study in $1997^{58}$ found that a combination of calculations and indices, such as oxygenation index (OI), arterial-to-alveolar oxygen tension ratio $\left(\mathrm{a} / \mathrm{A} \mathrm{PO}_{2}\right)$, and others, provided useful prognostic information when managing 47 very low-birth-weight infants. While they did not use a CDSS tool, it is clear that widespread use of such indices would benefit from computer support. Further work by van Kaam et al has shown the benefits of automated closed-loop control of inspired oxygen in premature infants. ${ }^{59}$ Unfortunately, this technology is not permitted in the United States yet.

\section{CDSS for Initiation of Mechanical Ventilation}

Some CDSS have been designed to assist with initial ventilator settings. ${ }^{52,60,61}$ While there are only limited reports of those CDSS tools, findings consistently show that CDSS-guided initiation results in comparable outcomes to cases in which ventilation was initiated by experts (vide supra). These sort of tools may be particularly useful in emergency situations or when less experienced clinicians need to initiate ventilator therapy.

\section{Weaning from Mechanical Ventilation}

While there are limited reports of pediatric CDSS tools for ventilator initiation, there have been more pediatric studies regarding CDSS support for weaning. Prolonged ventilation is associated with significant morbidity, hospital cost, psychosocial and physical risks to the child, and even death. ${ }^{62}$ This was also found to be true of failed extubations in PICU practice. ${ }^{63}$ Timely and effective weaning may reduce morbidity and mortality, ${ }^{16,64}$ whereas delays in weaning can promote further lung injury and contribute to patient discomfort and analgesic tolerance. ${ }^{16}$ To date, no consensus has been reached on explicit criteria that can be used to identify when patients are ready to wean or the best way to achieve weaning. ${ }^{18,65}$ A metaanalysis showed shorter time on the ventilator when management was guided by weaning protocols, but there was insufficient evidence to determine whether there was an overall benefit on clinical outcomes. ${ }^{62}$

Reports of weaning-focused CDSS in the PICU have been variable, ${ }^{16}$ with one study of protocol-managed ventilator weaning showing reduced time to extubation readiness ${ }^{66}$ and another study showing no decrease in weaning time. ${ }^{27}$ Both of these studies were limited to the weaning phase alone. A study that used a protocol for overall ventilator management in addition to weaning found reduced weaning time and time to spontaneous breathing modes but no difference in overall ventilator duration. ${ }^{16}$ That study used a paper protocol, and a limitation was their inability to determine compliance with the protocol. A larger study with 380 adults and older children in a Taiwanese respiratory care unit found reduced number of days on the ventilator and health care cost savings when weaning was managed with a CDSS system, noting that the CDSS identified earlier the readiness for weaning. ${ }^{67}$

\section{Conclusion}

The study of CDSS tools will help provide potential insights into optimal approaches for mechanical ventilation in the PICU. The search continues for more lung-protective approaches to mechanical ventilation. Best practices are a moving target. Mechanical ventilators continue to evolve, and there is evidence that current strategies may not be protective enough for all patients. ${ }^{4}$ Novel physiologic approaches are emerging, which, combined with advances in bioengineering, will continue to improve the delivery of mechanical ventilation. As large volumes of detailed real-time data become increasingly available, "smart monitoring," tools for prognosis, advice for targeted therapeutic approaches, and other forms of CDS will undoubtedly continue to emerge and evolve, providing a platform to support large-scale clinical trials, making those trials faster, more impactful, and less expensive to conduct. ${ }^{4}$

There is a pressing need for further technological development and for rigorous large scale studies of open- and closed-loop mechanical ventilation CDSS in pediatrics. ${ }^{13,56,68}$ Differences between adult and pediatric ICU practices, such as 
higher reliance in the PICU on noninvasive but continuous blood gas estimates (e.g., $\mathrm{Spo}_{2}$ ), ${ }^{1,48}$ highlight the need for pediatric-specific CDSS tools rather than direct adoption of adult ICU tools.

\section{References}

1 Khemani RG, Markovitz BP, Curley MA. Characteristics of children intubated and mechanically ventilated in 16 PICUs. Chest 2009; 136(3):765-771

2 Randolph AG. A practical approach to evidence-based medicine: lessons learned from developing ventilator management protocols. Crit Care Clin 2003;19(3):515-527

3 Randolph AG. How are children mechanically ventilated in pediatric intensive care units? Intensive Care Med 2004;30(5): 746-747

4 Slutsky AS. History of mechanical ventilation. From Vesalius to ventilator-induced lung injury. Am J Respir Crit Care Med 2015; 191(10):1106-1115

5 Hickling KG, Henderson SJ, Jackson R. Low mortality associated with low volume pressure limited ventilation with permissive hypercapnia in severe adult respiratory distress syndrome. Intensive Care Med 1990;16(6):372-377

6 The Acute Respiratory Distress Syndrome Network. Ventilation with lower tidal volumes as compared with traditional tidal volumes for acute lung injury and the acute respiratory distress syndrome. N Engl J Med 2000;342(18):1301-1308

7 Papazian L, Forel JM, Gacouin A, et al; ACURASYS Study Investigators. Neuromuscular blockers in early acute respiratory distress syndrome. N Engl J Med 2010;363(12):1107-1116

8 Briel M, Meade M, Mercat A, et al. Higher vs lower positive endexpiratory pressure in patients with acute lung injury and acute respiratory distress syndrome: systematic review and meta-analysis. JAMA 2010;303(9):865-873

9 Cortes GA, Marini JJ. Update: adjuncts to mechanical ventilation. Curr Opin Anaesthesiol 2012;25(2):156-163

10 Lyerla F, LeRouge C, Cooke DA, Turpin D, Wilson L. A nursing clinical decision support system and potential predictors of head-of-bed position for patients receiving mechanical ventilation. American journal of critical care: an official publication. American Association of Critical-Care Nurses 2010;19(1):39-47

11 Santschi M, Jouvet P, Leclerc F, et al; PALIVE Investigators; Pediatric Acute Lung Injury and Sepsis Investigators Network (PALISI); European Society of Pediatric and Neonatal Intensive Care (ESPNIC). Acute lung injury in children: therapeutic practice and feasibility of international clinical trials. Pediatr Crit Care Med 2010;11(6):681-689

12 de Jager P, Burgerhof JG, van Heerde M, Albers MJ, Markhorst DG, Kneyber MC. Tidal volume and mortality in mechanically ventilated children: a systematic review and meta-analysis of observational studies*. Crit Care Med 2014;42(12):2461-2472

13 Khemani RG, Newth CJ. The design of future pediatric mechanical ventilation trials for acute lung injury. Am J Respir Crit Care Med 2010;182(12):1465-1474

14 Sucher JF, Moore FA, Todd SR, Sailors RM, McKinley BA. Computerized clinical decision support: a technology to implement and validate evidence based guidelines. J Trauma 2008;64(2):520-537

15 Newth CJ. Respiratory disease and respiratory failure: implications for the young and the old. Br J Dis Chest 1986;80(3):209-217

16 Restrepo RD, Fortenberry JD, Spainhour C, Stockwell J, Goodfellow LT. Protocol-driven ventilator management in children: comparison to nonprotocol care. J Intensive Care Med 2004;19(5):274-284

17 Bernstein DB, Nguyen B, Allen GB, Bates JH. Elucidating the fuzziness in physician decision making in ARDS. J Clin Monit Comput 2013;27(3):357-363
18 Newth CJ, Venkataraman S, Willson DF, et al; Eunice Shriver Kennedy National Institute of Child Health and Human Development Collaborative Pediatric Critical Care Research Network. Weaning and extubation readiness in pediatric patients. Pediatr Crit Care Med 2009;10(1):1-11

19 The Pediatric Acute Lung Injury Consensus Conference Group. Pediatric acute respiratory distress syndrome: consensus recommendations from the Pediatric Acute Lung Injury Consensus Conference. Pediatr Crit Care Med 2015;16(5):428-439

20 Jouvet P, Hernert P, Wysocki M. Development and implementation of explicit computerized protocols for mechanical ventilation in children. Ann Intensive Care 2011;1(1):51

21 Morris AH. Treatment algorithms and protocolized care. Curr Opin Crit Care 2003;9(3):236-240

22 Khemani RG, Sward K, Morris A, Dean JM, Newth CJ; NICHD Collaborative Pediatric Critical Care Research Network (CPCCRN). Variability in usual care mechanical ventilation for pediatric acute lung injury: the potential benefit of a lung protective computer protocol. Intensive Care Med 2011;37(11):1840-1848

23 East TD, Heermann LK, Bradshaw RL, et al. Efficacy of computerized decision support for mechanical ventilation: results of a prospective multi-center randomized trial. Proc AMIA Symp 1999:251-255

24 Willson DF, Thomas NJ, Markovitz BP, et al; Pediatric Acute Lung Injury and Sepsis Investigators. Effect of exogenous surfactant (calfactant) in pediatric acute lung injury: a randomized controlled trial. JAMA 2005;293(4):470-476

25 Curley MA, Arnold JH, Thompson JE, et al; Pediatric Prone Positioning Study Group. Clinical trial design-effect of prone positioning on clinical outcomes in infants and children with acute respiratory distress syndrome. J Crit Care 2006;21(1):23-32, discussion 32-37

26 Jouvet P, Farges C, Hatzakis G, et al. Weaning children from mechanical ventilation with a computer-driven system (closedloop protocol): a pilot study. Pediatr Crit Care Med 2007;8(5): 425-432

27 Randolph AG, Wypij D, Venkataraman ST, et al; Pediatric Acute Lung Injury and Sepsis Investigators (PALISI) Network. Effect of mechanical ventilator weaning protocols on respiratory outcomes in infants and children: a randomized controlled trial. JAMA 2002; 288(20):2561-2568

28 Randolph AG, Clemmer TP, East TD, et al. Evaluation of compliance with a computerized protocol: weaning from mechanical ventilator support using pressure support. Comput Methods Programs Biomed 1998;57(3):201-215

29 Sward K, Newth CJ, Page K, Dean JM. Pediatric intensivist attitudes about a ventilator management computer protocol. Am J Respir Crit Care Med 2015;191:A4772

30 Morris AH. Developing and implementing computerized protocols for standardization of clinical decisions. Ann Intern Med 2000; 132(5):373-383

31 Petty J. Understanding neonatal ventilation: strategies for decision making in the NICU. Neonatal Netw 2013;32(4): 246-261

32 Eslami S, Abu-Hanna A, Schultz MJ, de Jonge E, de Keizer NF. Evaluation of consulting and critiquing decision support systems: effect on adherence to a lower tidal volume mechanical ventilation strategy. J Crit Care 2012;27(4):425.e1-425.e8

33 Eslami S, de Keizer NF, Abu-Hanna A, de Jonge E, Schultz MJ. Effect of a clinical decision support system on adherence to a lower tidal volume mechanical ventilation strategy. J Crit Care 2009;24(4): 523-529

34 Blagev DP, Hirshberg EL, Sward K, et al. The evolution of eProtocols that enable reproducible clinical research and care methods. J Clin Monit Comput 2012;26(4):305-317

35 Morris AH, Orme JJr, Truwit JD, et al. A replicable method for blood glucose control in critically Ill patients. Crit Care Med 2008;36(6): 1787-1795 
36 Morris AH, Hirshberg E, Sward KA. Computer protocols: how to implement. Best Pract Res Clin Anaesthesiol 2009;23(1):51-67

37 Sward K, Orme J Jr, Sorenson D, Baumann L, Morris AH; Reengineering Critical Care Clinical Research Investigators. Reasons for declining computerized insulin protocol recommendations: application of a framework. J Biomed Inform 2008;41(3):488-497

38 Bhutani VK. Clinical applications of pulmonary function and graphics. Semin Neonatol 2002;7(5):391-399

39 Gholami B, Bailey JM, Haddad WM, Tannenbaum AR. Clinical decision support and closed-loop control for cardiopulmonary management and intensive care unit sedation using expert systems. IEEE Trans Contr Syst Technol 2012;20(5):1343-1350

40 Sorenson D, Grissom CK, Carpenter L, et al; Reengineering Clinical Research in Critical Care Investigators. A frame-based representation for a bedside ventilator weaning protocol. J Biomed Inform 2008;41(3):461-468

41 Nguyen B, Bernstein DB, Bates JH. Controlling mechanical ventilation in acute respiratory distress syndrome with fuzzy logic. J Crit Care 2014;29(4):551-556

42 Karbing DS, Allerød C, Thomsen LP, et al. Retrospective evaluation of a decision support system for controlled mechanical ventilation. Med Biol Eng Comput 2012;50(1):43-51

43 Tehrani FT, Abbasi S. A model-based decision support system for critiquing mechanical ventilation treatments. J Clin Monit Comput 2012;26(3):207-215

44 Tzavaras A, Weller PR, Prinianakis G, Lahana A, Afentoulidis P, Spyropoulos B. Locating of the required key-variables to be employed in a ventilation management decision support system. Conf Proc IEEE Eng Med Biol Soc 2011;2011:112-115

45 Lee A, Mouhieddine M, Zekert M, Popow C. Computer determined compliance of the respiratory system (Crs) in ventilated newborn infants. Wien Klin Wochenschr 1994;106(7):193-196

46 Flechelles O, Ho A, Hernert P, et al. Simulations for mechanical ventilation in children: review and future prospects. Crit Care Res Pract 2013;2013:943281

47 Kretschmer J, Wahl A, Möller K. Dynamically generated models for medical decision support systems. Comput Biol Med 2011;41(10): 899-907

48 Khemani RG, Celikkaya EB, Shelton CR, et al. Algorithms to estimate $\mathrm{PaCO} 2$ and $\mathrm{pH}$ using noninvasive parameters for children with hypoxemic respiratory failure. Respir Care 2014;59(8): 1248-1257

49 Kelly AM, Klim S, Rees SE. Agreement between mathematically arterialised venous versus arterial blood gas values in patients undergoing non-invasive ventilation: a cohort study. Emerg Med J 2014;31(e1):e46-e49

50 Newth CJ, Sward K, Khemani RG, Page K, Dean JM. Variability in usual care oxygenation and ventilation decisions during mechanical ventilation for pediatric acute lung injury. Am J Respir Crit Care Med 2015;191:314

51 Newth CJ, Sward K, Khemani RG, Page K, Dean JM; Eunice Kennedy Shriver NICHD Collaborative Pediatric Critical Care Network Investigators. Variability in usual care mechanical ventilation for pediatric acute lung injury. Am J Respir Crit Care Med 2014;189: A2613

52 Laubscher TP, Frutiger A, Fanconi S, Jutzi H, Brunner JX. Automatic selection of tidal volume, respiratory frequency and minute ventilation in intubated ICU patients as start up procedure for closed-loop controlled ventilation. Int J Clin Monit Comput 1994; 11(1):19-30

53 Claure N, Gerhardt T, Hummler H, Everett R, Bancalari E. Computer-controlled minute ventilation in preterm infants undergoing mechanical ventilation. J Pediatr 1997;131(6):910-913

54 Jouvet P, Eddington A, Payen V, et al. A pilot prospective study on closed loop controlled ventilation and oxygenation in ventilated children during the weaning phase. Crit Care 2012;16(3):R85

55 Blackwood B, Junk C, Lyons JD, McAuley DF, Rose L. Role responsibilities in mechanical ventilation and weaning in pediatric intensive care units: a national survey. American journal of critical care: an official publication. American Association of Critical-Care Nurses 2013;22(3):189-197

56 Rose L, Schultz MJ, Cardwell CR, Jouvet P, McAuley DF, Blackwood B. Automated versus non-automated weaning for reducing the duration of mechanical ventilation for critically ill adults and children. Cochrane Database Syst Rev 2013;6:CD009235

57 Carlo WA, Pacifico L, Chatburn RL, Fanaroff AA. Efficacy of computer-assisted management of respiratory failure in neonates. Pediatrics 1986;78(1):139-143

58 Despotova-Toleva L, Petrov A. Feasibility for evaluation of the efficacy of conventional ventilatory support in very low birth weight infants. Folia Med (Plovdiv) 1997;39(4):55-64

59 van Kaam AH, Hummler HD, Wilinska M, et al. Automated versus manual oxygen control with different saturation targets and modes of respiratory support in preterm infants. J Pediatr 2015; 167(3):545-550.e2

60 Akbulut FP, Akkur E, Akan A, Yarman BS. A decision support system to determine optimal ventilator settings. BMC Med Inform Decis Mak 2014;14:3

61 Allerød C, Rees SE, Rasmussen BS, et al. A decision support system for suggesting ventilator settings: retrospective evaluation in cardiac surgery patients ventilated in the ICU. Comput Methods Programs Biomed 2008;92(2):205-212

62 Blackwood B, Murray M, Chisakuta A, Cardwell CR, O'Halloran P. Protocolized versus non-protocolized weaning for reducing the duration of invasive mechanical ventilation in critically ill paediatric patients. Cochrane Database Syst Rev 2013;7:CD009082

63 Kurachek SC, Newth CJ, Quasney MW, et al. Extubation failure in pediatric intensive care: a multiple-center study of risk factors and outcomes. Crit Care Med 2003;31(11):2657-2664

64 Bouadma L, Lellouche F, Cabello B, et al. Computer-driven management of prolonged mechanical ventilation and weaning: a pilot study. Intensive Care Med 2005;31(10):1446-1450

65 Leclerc F, Noizet O, Botte A, et al. [Weaning from invasive mechanical ventilation in pediatric patients (excluding premature neonates)]. Arch Pediatr 2010;17(4):399-406

66 Schultz TR, Lin RJ, Watzman HM, et al. Weaning children from mechanical ventilation: a prospective randomized trial of protocol-directed versus physician-directed weaning. Respir Care 2001; 46(8):772-782

67 Hsu JC, Chen YF, Chung WS, Tan TH, Chen T, Chiang JY. Clinical verification of a clinical decision support system for ventilator weaning. Biomed Eng Online 2013;12(Suppl 1):S4

68 Kacmarek RM. The mechanical ventilator: past, present, and future. Respir Care 2011;56(8):1170-1180 